\title{
Quarkyonic phase in the strong coupling region of lattice QCD
}

\author{
Akira Ohnishi* and Kohtaroh Miura \\ Yukawa Institute for Theoretical Physics, Kyoto University, Kyoto 606-8502, Japan \\ E-mail: ohnishi@yukawa.kyoto-u.ac.jp
}

\begin{abstract}
QCD phase diagram is studied at finite temperature $(T)$ and chemical potential $(\mu)$ in the strong coupling lattice QCD including finite coupling $\left(1 / g^{2}\right)$ effects for color $\mathrm{SU}\left(N_{c}\right)$. We find that there appears a phase, where the chiral symmetry is weakly but spontaneously broken and the baryon density is high, at $N_{c}=3$ with one species of staggered fermions $\left(N_{f}=4\right)$. This phase may correspond to the quarkyonic (QY) phase suggested at large $N_{c}$. The phase transition is found to be the first and second order for the hadron (Nambu-Goldstone) to QY and QY to Wigner phase, respectively. The QY phase appears as a result of interplay of two order parameters, the chiral condensate and the repulsive vector field for quarks, the latter of which is found to appear from the temporal plaquette and leads to the suppression of the effective chemical potential at high densities.
\end{abstract}

The XXVI International Symposium on Lattice Field Theory

July 14-19 2008

Williamsburg, Virginia, USA

\footnotetext{
*Speaker.
} 


\section{Introduction}

What is the next to the hadronic Nambu-Goldstone (NG) phase in the larger $\mu$ direction? Baryon rich QGP, Color SuperConductor, or other form of matter? This is one of the central questions in dense QCD, and is relevant to dense matter in nature, which would be realized, for example, in neutron star core or during during black hole formation. Since Monte-Carlo (MC) simulations are not yet reliable in the region $\mu / T>1$, then it is necessary to invoke some approximations in QCD or effective models of QCD.

Recently, McLerran and Pisarski have shown that the next phase at large $N_{c}$ should be the so called quarkyonic (QY) phase, in which the colors are confined and the baryon density is high [1]. Very recently, QY is also discussed at $N_{c}=3$ in effective models of QCD [2] 3]. MC results also show the transition to high density phase [4], but its nature is not yet known. It is now very important and urgently required to discuss the possibility of QY in QCD for $N_{c}=3$.

In this proceedings, we investigate the phase diagram in the strong coupling lattice QCD (SCLQCD) [5, 6, 7, 8, , which is another powerful tool in studying dense matter. We take account of the finite coupling effects in the order of $1 / g^{2}$ [8, 9 , and introduce an auxiliary field representing the quark number density $\rho_{q}$ as an order parameter in addition to the chiral condensate [10]. This multi-order parameter treatment is essential in understanding QY.

\section{Effective potential in strong coupling lattice QCD}

We start from the lattice action with one species of staggered fermions,

$$
S_{\mathrm{LQCD}}=\frac{1}{2} \sum_{x, v}\left[\eta_{v, x} \bar{\chi}_{x} U_{v, x} \chi_{x+\hat{v}}-\eta_{v, x}^{-1} \bar{\chi}_{x+\hat{v}} U_{v, x}^{\dagger} \chi_{x}\right]-\frac{1}{g^{2}} \sum_{\square} \operatorname{tr}\left[U_{\square}+U_{\square}^{\dagger}\right]+m_{0} \sum_{x} \bar{\chi}_{x} \chi_{x},
$$

where $\eta_{v, x}$ represents the staggered factor combined with the lattice chemical potential, $\left(\eta_{0, x}, \eta_{j, x}\right)=$ $\left(e^{\mu},(-1)^{x_{0}+\cdots+x_{j-1}}\right)$, and the partition function is given as, $\mathscr{Z}=\int \mathscr{D}[U, \chi, \bar{\chi}] \exp \left[-S_{\mathrm{LQCD}}\right]$. We consider the leading $\left(S_{\mathrm{SCL}}\right)$ [5, 6, 7 and the next-to-leading order terms \&, 9 in the strong coupling $\left(1 / g^{2}\right)$ expansion, $S=S_{\mathrm{SCL}}+\Delta S_{\beta}^{(\tau)}+\Delta S_{\beta}^{(s)}+\mathscr{O}\left(1 / \sqrt{d}, 1 / g^{4}\right)$, where $d=3$ is the spatial dimension. NLO terms $\left(\Delta S_{\beta}^{(\tau, s)}\right)$ are generated from spatial link integrals of plaquettes multiplied by the corresponding fermion terms. For example, temporal plaquette contribution is given as,

$$
\begin{aligned}
\Delta S_{\beta}^{(\tau)} & =\frac{1}{4 N_{c}^{2} g^{2}} \sum_{x, j>0}\left(V_{x}^{+} V_{x+\hat{j}}^{-}+V_{x}^{+} V_{x-\hat{j}}^{-}\right), \\
V_{x}^{+} & =e^{\mu} \bar{\chi}_{x} U_{0}(x) \chi_{x+\hat{0}}, \quad V_{x}^{-}=e^{-\mu} \bar{\chi}_{x+\hat{0}} U_{0}^{\dagger}(x) \chi_{x} .
\end{aligned}
$$

In the standard SCL prescriptions, we next introduce auxiliary fields in order to reduce the power of fermion fields down to bilinear terms. Since we have products of different types of composites $\left(V^{+}\right.$and $\left.V^{-}\right)$in Eq. 2.2), the standard Hubbard-Stratonovich transformation is insufficient. Here we propose an extended Hubbard-Stratonovich (EHS) transformation where two auxiliary fields are introduced [10],

$$
\begin{aligned}
e^{\alpha A B} & =\int d \varphi d \phi e^{-\alpha\left\{\phi^{2}-(A+B) \varphi+\phi^{2}-i(A-B) \phi\right\}} \\
& =\left.\int d \psi d \bar{\psi} e^{-\alpha\{\bar{\psi} \psi-A \psi-\bar{\psi} B\}} \approx e^{-\alpha\{\bar{\psi} \psi-A \psi-\bar{\psi} B\}}\right|_{\text {stationary }},
\end{aligned}
$$


where $\psi=\varphi+i \phi$ and $\bar{\psi}=\varphi-i \phi$. This EHS transformation has a merit that we can keep the invariance under the transformation, $A \rightarrow \lambda A$ and $B \rightarrow \lambda^{-1} B$, even after the stationary condition approximation by assuming $\psi$ and $\bar{\psi}$ transform independently, $\psi \rightarrow \lambda^{-1} \psi$ and $\bar{\psi} \rightarrow \lambda \bar{\psi}$. Since stationary values of $\varphi$ and $\phi$ can be complex, stationary values of $\psi$ and $\bar{\psi}$ are not necessarily complex conjugate. Specifically when both $\langle A\rangle$ and $\langle B\rangle$ are real, the stationary value of $\phi$ is pure imaginary, and we obtain the following relation,

$$
e^{\alpha A B} \approx e^{-\alpha\left\{\varphi^{2}-(A+B) \varphi-\phi^{2}+(A-B) \phi\right\}} .
$$

This corresponds to the mean field and saddle point approximation for $\varphi$ and $\phi$, respectively in Eq. (2.4). provided that the stationary value of $\phi$ is pure imaginary, $\phi \rightarrow i \phi$. It also corresponds to substituting $\psi=\varphi+\phi$ and $\bar{\psi}=\varphi-\phi$ in Eq. 2.5.

For the temporal plaquette action $\Delta S_{\beta}^{(\tau)}$, we substitute $(\alpha, A, B)=\left(1 / 4 N_{c}^{2} g^{2},-V_{x}^{+}, V_{x+\hat{j}}^{-}\right)$, and obtain,

$$
\Delta S_{\beta}^{(\tau)} \approx \frac{1}{4 N_{c}^{2} g^{2}} \sum_{x, j>0}\left[\varphi_{\tau}^{2}+\left(V_{x}^{+}-V_{x+\hat{j}}^{-}\right) \varphi_{\tau}-\phi_{\tau}^{2}-\left(V_{x}^{+}+V_{x+\hat{j}}^{-}\right) \phi_{\tau}\right]+(j \leftrightarrow-j) .
$$

Combined with the temporal hopping term in the QCD lattice action Eq. 2.1), we obtain

$$
\begin{aligned}
\frac{1}{2} \sum_{x}\left(V_{x}^{+}-V_{x}^{-}\right)+\Delta S_{\beta}^{(\tau)}= & \frac{1}{2}\left(1+\beta_{\tau} \varphi_{\tau}\right) \sum_{x}\left(e^{\mu-\beta_{\tau} \phi_{\tau}} \bar{\chi}_{x} U_{0}(x) \chi_{x+\hat{0}}-e^{-\mu+\beta_{\tau} \phi_{\tau}} \bar{\chi}_{x+\hat{0}} U_{0}^{\dagger}(x) \chi_{x}\right) \\
& +N_{\tau} L^{d} \frac{\beta_{\tau}}{2}\left(\varphi_{\tau}^{2}-\phi_{\tau}^{2}\right),
\end{aligned}
$$

where $N_{\tau}$ and $L$ denote the temporal and spatial lattice sizes, respectively, $\beta_{\tau}=d / N_{c}^{2} g^{2}$, and we have assumed that the auxiliary fields $\varphi_{\tau}$ and $\phi_{\tau}$ are constant. We find that the saddle point field $\phi_{\tau}$ modifies the chemical potential effectively, $\tilde{\mu}=\mu-\beta_{\tau} \phi_{\tau}$. As discussed later, $\phi_{\tau}$ corresponds to the quark number density, and plays the role of another order parameter characterizing dense matter.

We also obtain the effective action for the spatial plaquette contribution $\Delta S_{\beta}^{(s)}$ in a similar way, except that the saddle point field $\phi_{s}$ is found to give no effects. In the same procedure as that in SCL, we introduce the auxiliary field $\sigma$ for the chiral condensate, and perform the temporal link integral of the quark determinant, resulting in the effective potential, $\mathscr{F}_{\text {eff }}\left(\sigma, \varphi_{\tau}, \phi_{\tau}, \varphi_{s} ; \mu, T\right)$. By substituting equilibrium values of $\varphi_{\tau}$ and $\varphi_{s}$, the effective potential $\mathscr{F}_{\text {eff }}\left(\sigma, \phi_{\tau} ; \mu, T\right)$ is found to be [10],

$$
\begin{aligned}
\mathscr{F}_{\mathrm{eff}} & =\mathscr{F}_{\mathrm{X}}\left(\sigma, \phi_{\tau}\right)+\mathscr{V}_{\mathrm{q}}\left(m_{q}(\sigma), \tilde{\mu}\left(\phi_{\tau}\right), T\right), \\
\mathscr{F}_{\mathrm{X}} & =\frac{1}{2} b_{\sigma} \sigma^{2}+\frac{\beta_{\tau}}{2} \sigma^{2}\left(m_{q}^{\mathrm{SCL}}\right)^{2}+\frac{3 d \beta_{s}}{2} \sigma^{4}-\frac{\beta_{\tau}}{2} \phi_{\tau}^{2}, \\
\mathscr{V}_{\mathrm{q}} & =-T \log \left[X_{N_{c}}\left(E_{q} / T\right)+2 \cosh \left(N_{c} \tilde{\mu} / T\right)\right], \\
m_{q} & =m_{q}^{\mathrm{SCL}}\left(1-N_{c} \beta_{\tau}\right)+\beta_{\tau} \sigma\left(m_{q}^{\mathrm{SCL}}\right)^{2}+2 d \beta_{s} \sigma^{3},
\end{aligned}
$$

where $\beta_{s}=(d-1) / 8 N_{c}^{4} g^{2}, b_{\sigma}=d / 2 N_{c}, m_{q}^{\mathrm{SCL}}=b_{\sigma} \sigma+m_{0}, X_{N}(x)=\sinh [(N+1) x] / \sinh x$, and $E_{q}=\operatorname{arcsinh}\left(m_{q}\right)$. We have omitted higher order terms of $\mathscr{O}\left(1 / g^{4}\right)$ in $\mathscr{F}_{X}, m_{q}$ and $\tilde{\mu}$. We search for $\sigma$ which minimizes $\mathscr{F}_{\text {eff }}$ under the saddle point constraint for $\phi_{\tau}, \partial \mathscr{F}_{\text {eff }} / \partial \phi_{\tau}=0$, which reads, $\phi_{\tau}=-\partial \mathscr{V}_{\mathrm{q}} / \partial \tilde{\mu}=-\partial \mathscr{F}_{\text {eff }} / \partial \mu=\rho_{q}$. Since $\rho_{q}$ contains $\phi_{\tau}$ via $\tilde{\mu}$, we need to solve this constraint 


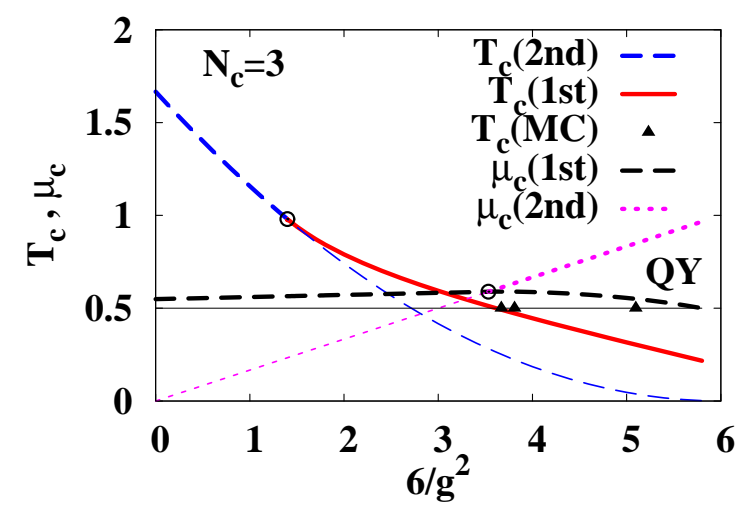

Figure 1: Coupling dependence of critical temperature and chemical potential. Results with $N_{c}=3$ in the chiral limit are shown in the lattice unit.

in a self-consistent manner. This is one of the main differences of the present treatment from those in previous works $\llbracket \mathbb{8}$, where $\mathscr{V}_{\mathrm{q}}$ is also expanded in $1 / g^{2}$ via the dependence in $m_{q}$ and $\tilde{\mu}$.

The auxiliary field $\phi_{\tau}$ may be interpreted as a repulsive vector field for quarks. In relativistic mean field (RMF) models of nuclei [11], the isoscalar-vector field $\omega$ contributes to the energy density as, $\varepsilon_{V}=-m_{\omega}^{2} \omega^{2} / 2+g_{\omega} \rho_{B}\left(\tilde{\mu}_{B}\right) \omega+\ldots$, where $\omega$ is the temporal component of the omega meson field $\omega^{v}$. The negative coefficient of $\omega^{2}$ results in the repulsive interaction in nuclei, and the coupling to $\rho_{B}$ leads to the shift of $\mu_{B}$ as, $E+g_{\omega} \omega-\mu_{B}=E-\left(\mu_{B}-g_{\omega} \omega\right)=E-\tilde{\mu}$. The saddle point constraint gives $\omega \propto \rho_{B}$. All of these characters apply to the auxiliary field $\phi_{\tau}$ in SC-LQCD.

\section{Results}

At finite coupling, the hadron phase is known to be compressed in the temperature direction [9, 12]. The decrease of the second order critical temperature is mainly caused by the temporal scale factor $\left(1+\beta_{\tau} \varphi_{\tau}\right)$ in Eq. (2.8), which has a similar effect to the temporal lattice spacing modification. The suppression of $T_{c}$ would be a natural consequence of finite coupling, since hadrons are less bound than in SCL. The high $T$ transition becomes the first order in the region $6 / g^{2}>1.40$. This change is caused by the higher order terms of $\sigma$ in $\mathscr{F}_{\mathrm{X}}$ and $m_{q}$. These terms give rise to a large energy gain in $\mathscr{F}_{\text {eff }}$ and generate a local minimum at large $\sigma$. The calculated critical temperature seems to be in agreement with the MC results with $N_{\tau}=2$ at strong coupling [13]. The critical coupling with $N_{\tau}=2$ is $\beta_{c}=6 / g_{c}^{2}=5.097(1)$ [14 in a quenched calculation $\left(m_{0}=\infty\right)$, and it decreases to $\beta_{c}=3.81(2)\left(m_{0}=0.05\right)$ and $\beta_{c}=3.67(2)\left(m_{0}=0.025\right)$ [13 on a $8^{3} \times 2$ lattice with one species of staggered fermions $\left(N_{f}=4\right)$. Thus MC results with smaller quark masses become close to the present result $\beta_{c}=3.61$ at $T_{c}=1 / N_{\tau}=1 / 2$ in the chiral limit.

Finite coupling effects also modifies the critical chemical potential. From the condition $\partial^{2} \mathscr{F}_{\text {eff }} / \partial \sigma^{2}=$ 0 at $\sigma=0$, the second order critical chemical potential is obtained as,

$$
\mu_{c}^{(2 n d)}=\tilde{\mu}_{c}^{(2 n d)}+\beta_{\tau} \rho_{q}\left(\sigma=0 ; \tilde{\mu}_{c}^{(2 n d)}, T\right), \quad \tilde{\mu}_{c}^{(2 n d)}=\frac{T}{N_{c}} \operatorname{arccosh}\left[1+\left(N_{c}+3\right)\left(T_{c}^{(2 n d)}-T\right) / 2 T\right] .
$$



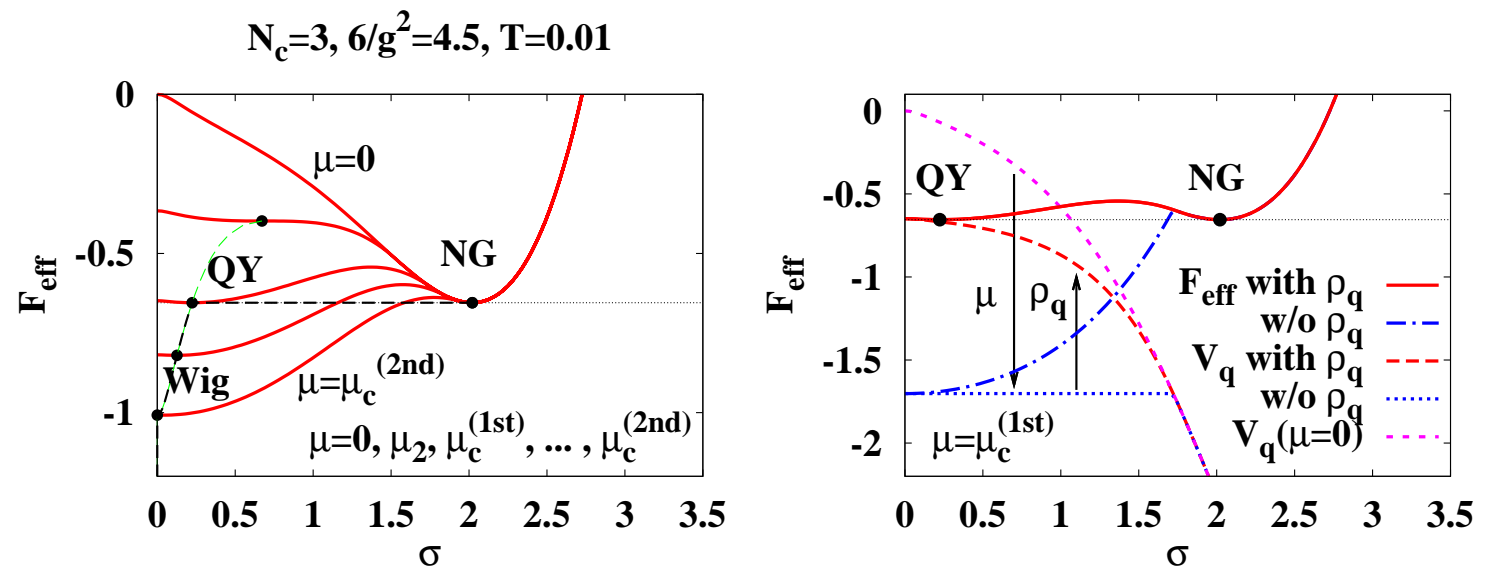

Figure 2: Upper panel: Effective potential $\mathscr{F}_{\text {eff }}$ as a function of $\sigma$ at $\mu=0, \mu_{2}$ (the smallest $\mu$ at which two local minima appear), $\mu_{c}^{(1 s t)},\left(\mu_{c}^{(1 s t)}+\mu_{c}^{(2 n d)}\right) / 2$ and $\mu_{c}^{(2 n d)}$. Lower panel: The quark number density $\rho_{q}$ and chemical potential $\mu$ effects on the interaction term $\mathscr{V}_{\mathrm{q}}$. We show the results in the case of $N_{c}=3,6 / g^{2}=$ $4.5, T=0.01$ in the lattice unit.
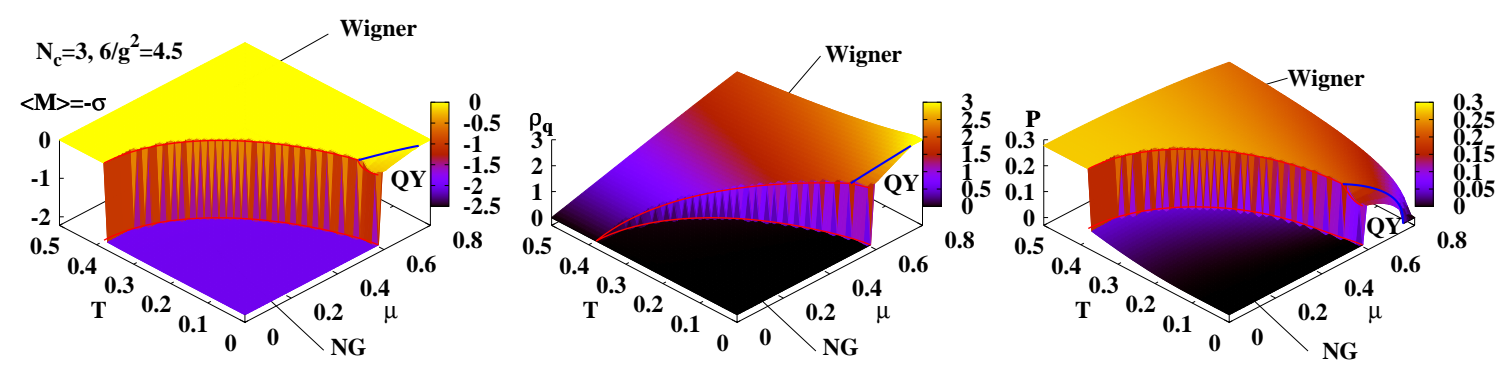

Figure 3: Chiral condensate $\langle\bar{\chi} \chi\rangle=-\sigma$ (left), quark number density $\rho_{q}$ (middle), and Polyakov loop $P$ (right) as functions of $(\mu, T)$ with $N_{c}=3,6 / g^{2}=4.5$ in the chiral limit.

In SCL, we do not have the second term in Eq. (3.1) and $\mu_{c}^{(2 n d)}$ approaches zero at small $T$. As a result, we do not see the second order phase transition in cold matter in SCL. At finite coupling, the second term in Eq. (3.1) increases as the coupling decreases, and we have the region $\mu_{c}^{(1 s t)}<\mu<$ $\mu_{c}^{(2 n d)}$ at low $T$ at $6 / g^{2}>3.53$. In this region, the chiral symmetry is weakly but spontaneously broken, and the baryon density is finite. In the left panel of Fig. 2. we show the effective potential as a function of $\sigma$ at $T=0.01$. (Coupling constant is chosen to be $6 / g^{2}=4.5$ as a typical example with $\mu_{c}^{(1 s t)}<\mu_{c}^{(2 n d)}$.) From the Nambu-Goldstone phase $\left(\sigma \approx \sigma_{v a c}\right)$ at $\mu=0, \sigma$ jumps to a smaller value at $\mu=\mu_{c}^{(1 s t)}$, and reaches zero at $\mu=\mu_{c}^{(2 n d)}$, where the chiral restored (Wigner) phase is realized. In terms of the effective potential, two local minimum structure in $\sigma>0$ region comes from the suppression of effective chemical potential $\tilde{\mu}$ by the density $\left(\rho_{q}\right)$ effects. In another word, it comes from the repulsive effect of $\phi_{\tau}$ at small $\sigma$. While the interaction term $\mathscr{V}_{\mathrm{q}}$ in SCL stays almost constant in the region $E_{q}(\sigma)<\mu$ at low $T, \mathscr{V}_{\mathrm{q}}$ with $\rho_{q}$ effects is more repulsive at small $\sigma$ as shown in the right panel of Fig. 2

In Fig. 3, we show the chiral condensate $\langle\bar{\chi} \chi\rangle=-\sigma$ and the quark number density $\rho_{q}$ as functions of $(\mu, T)$. The phase diagram in SC-LQCD at finite coupling is shown in Fig. 4. In addition 


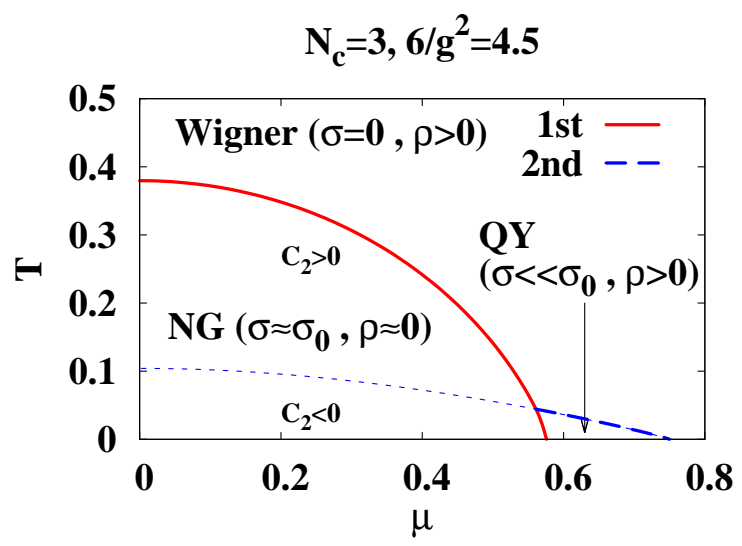

Figure 4: Phase diagram in SC-LQCD with $N_{c}=3,6 / g^{2}=4.5$

to the chiral broken Nambu-Goldstone (NG) phase $\left(\sigma \approx \sigma_{0}\right)$ and the chiral restored Wigner phase $(\sigma=0)$, a weakly chiral broken high density phase $\left(\sigma \ll \sigma_{0}, \rho_{q}>0\right)$, is found in a moderate chemical potential region at low $T$, which sits next to the hadronic NG phase in the larger $\mu$ direction. Similar results are also found in the PNJL model [3].

In the right panel of Fig. 3, we show the Polyakov loop $P$, which is evaluated by using the determinant technique [6] as,

$$
P \equiv \frac{1}{2 N_{c}}\left\langle\operatorname{tr}\left[\prod_{\tau} U_{0}+\prod_{\tau} U_{0}^{\dagger}\right]\right\rangle=\frac{X_{N_{c}-1} \cosh [\tilde{\mu} / T]+X_{1} \cosh \left[\left(N_{c}-1\right) \tilde{\mu} / T\right]}{N_{c}\left(X_{N_{c}}+2 \cosh \left[N_{c} \tilde{\mu} / T\right]\right)} .
$$

The Polyakov loop shown here is not large, e.g. $P(\sigma=\mu=0)=\left(N_{c}+2\right) /\left[N_{c}\left(N_{c}+3\right)\right]$, since it is not generated by the gluon dynamics, but driven by the quark determinant. The Polyakov loop is further suppressed in the weakly chiral broken high density phase compared to the chiral restored Wigner phase. Specifically, we find $P \rightarrow 1 / 2 N_{c}$ at large $N_{c}$ and $T=0$, which is half of that in the Wigner phase at $\mu=0$. It should be noted that the vanishing $P$ at $\mu>N_{c} \beta_{\tau}$ is the lattice artifact since it comes from the saturation, $\rho_{q}=N_{c}$.

The weakly chiral broken high density phase found in SC-LQCD can be regarded as the quarkyonic phase (QY) suggested at large $N_{c}$; it sits next to the hadronic NG phase in the $\mu$ direction, the density is high as $\mathscr{O}\left(N_{c}\right)$, and the Polyakov loop disappears at large $N_{c}$. Namely, it would evolve to a confined high density phase next to the hadronic phase at large $N_{c}$.

\section{Summary}

We have investigated the QCD phase diagram in the strong coupling lattice QCD including the finite coupling $\left(1 / g^{2}\right)$ effects, and found a weakly but spontaneously chiral broken high density phase to appear in the higher density region next to the hadronic Nambu-Goldstone phase in the strong coupling lattice QCD with finite coupling corrections at 6/ $/ g^{2}>3.53$ [10]. This phase can be regarded as the quarkyonic phase suggested at large $\left.N_{c} \llbracket 1\right]$, since the density is high $\left(\mathscr{O}\left(N_{c}\right)\right)$ and the color is confined at large $N_{c}$ in the sense that the Polyakov loop disappears. The transition from the hadronic NG to the quarkyonic phase is the first order, and that from the quarkyonic to the chiral 
restored Wigner phase is the second order. The quarkyonic phase appears as a result of interplay of two order parameters, the chiral condensate and the repulsive vector field for quarks, the latter of which is found to appear from the temporal plaquette in the extended Hubbard-Stratonovich transformation and leads to the suppression of the effective chemical potential at high densities.

There are several points to be improved in the present analysis; it has been carried out with one species of staggered fermions, which corresponds to $N_{f}=4$, in the next to leading order of the strong coupling expansion, $\mathscr{O}\left(1 / g^{2}\right)$, and in the leading order of the $1 / d$ expansion, $\mathscr{O}\left(d^{0}\right)$, where the baryonic composite effects [7, 15] are not included. Competition with the color superconducting (CSC) phase and comparison with the MC results at finite baryon density [4] are other interesting subjects to be investigated.

We would like to thank Prof. Larry McLerran, Dr. Kenji Fukushima, Prof. Noboru Kawamoto, Prof. Philippe de Forcrand, Dr. Michael Fromm, Dr. Barak Bringoltz, and Prof. Benjamin Svetitsky for useful discussions. This work is supported in part by KAKENHI 17070002 (Priority area) and 19540252, and the Yukawa International Program for Quark-hadron Sciences (YIPQS).

\section{References}

[1] L. McLerran and R. D. Pisarski, Nucl. Phys. A 796, 83 (2007).

[2] L. Y. Glozman and R. F. Wagenbrunn, Phys. Rev. D 77, 054027 (2008).

[3] K. Fukushima, Phys. Rev. D 77, 114028 (2008) [arXiv:0803.3318 [hep-ph]]; H. Abuki, R. Anglani, R. Gatto, G. Nardulli and M. Ruggieri, arXiv:0805.1509 [hep-ph].

[4] Z. Fodor, S. D. Katz and C. Schmidt, JHEP 0703, 121 (2007); P. de Forcrand and S. Kim, Phys. Lett. B 645, 339 (2007); P. de Forcrand and O. Philipsen, Nucl. Phys. B 673, 170 (2003); M. Fromm and $\mathrm{Ph}$. de Forcrand, in this proceedings.

[5] N. Kawamoto and J. Smit, Nucl. Phys. B 192, 100 (1981); P. H. Damgaard, N. Kawamoto, K. Shigemoto, Phys. Rev. Lett. 53, 2211 (1984); K. Fukushima, Prog. Theor. Phys. Suppl. 153, 204 (2004);

[6] Y. Nishida, Phys. Rev. D 69, 094501 (2004).

[7] N. Kawamoto, K. Miura, A. Ohnishi, T. Ohnuma, Phys. Rev. D 75, 014502 (2007).

[8] G. Faldt and B. Petersson, Nucl. Phys. B 265, 197 (1986);

N. Bilić, F. Karsch, K. Redlich, Phys. Rev. D 45, 3228 (1992).

[9] A. Ohnishi, N. Kawamoto and K. Miura, J. Phys. G 34, S655 (2007); A. Ohnishi, N. Kawamoto, K. Miura, K. Tsubakihara and H. Maekawa, Prog. Theor. Phys. Suppl. 168, 261 (2007).

[10] K. Miura and A. Ohnishi, arXiv:0806.3357 [nucl-th].

[11] B. D. Serot and J. D. Walecka, Adv. Nucl. Phys. 16, 1 (1986);

K. Tsubakihara and A. Ohnishi, Prog. Theor. Phys. 117, 903 (2007).

[12] N. Bilić and J. Cleymans, Phys. Lett. B 355, 266 (1995).

[13] P. de Forcrand, private communication.

[14] A. D. Kennedy, J. Kuti, S. Meyer and B. J. Pendleton, Phys. Rev. Lett. 54, 87 (1985).

[15] B. Bringoltz and B. Svetitsky, Phys. Rev. D 68 (2003) 034501 [arXiv:hep-lat/0211018]. 